\title{
Spherical indentation response of a Ni double gyroid nanolattice
}

\section{R.K. Prusty ${ }^{1}$, R.L. Narayan ${ }^{2}$, M. Scherer ${ }^{3}$, U. Steiner ${ }^{4}$, V.S. Deshpande ${ }^{5}$, N.A. Fleck ${ }^{5}$, U. Ramamurty $^{6 *}$}

${ }^{1}$ Department of Metallurgical and Materials Engineering, National Institute of Technology, Rourkela - 769008, India.

${ }^{2}$ Department of Materials Science and Engineering, Indian Institute of Technology, Delhi110016, India.

${ }^{3}$ Papierfabrik Louisenthal GmbH, Louisenthal 1, D-83703 Gmund am Tegernsee, Germany

${ }^{4}$ Adolphe Merkle Institute, Chemin des Verdiers, CH-1700 Fribourg, Switzerland

${ }^{5}$ Department of Engineering, University of Cambridge, Trumpington Street, Cambridge CB2

1PZ, UK

${ }^{6}$ School of Mechanical and Aerospace Engineering, Nanyang Technological University, Singapore 639798.

*Corresponding author; e-mail: uram@ntu.edu.sg

\begin{abstract}
The effect of indentation strain $\varepsilon_{\mathrm{i}}$ upon hardness $H$ and elastic modulus $E$ of a Ni Double Gyroid (DG) nanolattice was investigated using a spherically-tipped nanoindenter. $H$ remains invariant, while $E$ decreases linearly, with increasing $\varepsilon_{\mathrm{i}}$. Results reveal the progressive collapse of the DG lattice beneath the indenter. The measured values of $H$ and extrapolated value of $E$ at $\varepsilon_{\mathrm{i}}=0$ were used to estimate the yield strength and elastic modulus of the Ni cell walls. The latter was compared with the ideal strength of $\mathrm{Ni}$, nanocrystalline films and of sub-100 nm diameter single crystals.
\end{abstract}

Keywords: Cellular material; Nanoindentation; Hardness; Plastic deformation; Elastic modulus. 
Cellular metals range from metallic foams, of millimetre-cell size, to nanoporous metals with ligament and pore sizes in the nanometer ( $\mathrm{nm}$ ) scale; they are of interest from both scientific and technological perspectives. One such nanoporous metal lattice with the double gyroid (DG) architecture is the subject of this study. The lattices of DGs, as described in Ref. [1], have a unique but a well defined arrangement of ligaments and pores and are synthesized by the following method [2]. A DG template, over which the desired metal will be deposited, is made using selfassembling block copolymers [3]. Electroless plating of the target metal is then performed on over the template $[4,5]$. The volume fraction of the DG polymer template controls the relative density and pore volume of the final metal DG lattice. The polymer template is then selectively removed either by selective dissolution, hydrolysis [3], or the pyrolysis techniques [6], so as to obtain a free standing metal DG lattice. Such DG lattices have several applications where a high surface areavolume ratio is desired, such as the design of high performance electrochemical actuators [7].

Another important property that is relevant for a DG's performance and reliability is its structural integrity, which necessiates a knowledge of their mechanical properties and deformation behavior. The specific strength and stiffness of nanoporous metals can be significantly higher than their bulk counterparts, with the higher strength often attributed to the fact that the characteristic length is on a nm-scale [8-10]. This is because, structural components that correspond to these dimensions do not contain sufficient number of dislocations and require homogeneous nucleation of dislocation to initiate yielding [11,12]. The effective macroscopic strength of these materials is also dependent upon the architecture and connectivity of the constituent structural elements $[13,14]$. Recently, the strength of the cell walls in a Ni DG nanolattice was estimated from nanoindentation experiments using a Berkovich tip: the inferred strength of the cell walls approached the theoretical, ideal strength of pure $\mathrm{Ni}$ [15]. The extreme value of cell wall strength was rationalized by recourse to arguments on size effects on strength.

In the present study, we examine the effect of indentation strain upon the mechanical response of the Ni DG. This was accomplished by using a spherical tip indenter during nanoindentation. The ability to vary $\varepsilon_{\mathrm{i}}$ systematically in spherical indentation allows for the probing of the mechanical behavior of the cellular material in the undeformed state by extrapolation of the experimental data to the limit $\varepsilon_{\mathrm{i}}=0$. This facilitates a determination of the strength enhancement due to nanostructuring, which is the main objective of this study. In contrast, 
Khaderi et al [15] used a Berkovich indenter of geometrically self-similar head-shape; consequently, the average indentation strain $\varepsilon_{\mathrm{i}}$ was constant regardless of indent size.

Pure Ni DG films, of diameter $1 \mathrm{~mm}$ and thickness $2 \mu \mathrm{m}$, were deposited on a fluorinedoped tin oxide (FTO) coated glass substrate using a block co-polymer self-assembly technique, see Scherer et al. [3] for the details of this technique. The relative density of the specimens is $\rho_{\mathrm{r}}=$ 0.40 , as defined by the ratio of the density $\rho_{\text {DG }}$ of the Ni DG to the density $\rho_{\mathrm{B}}$ of solid $\mathrm{Ni}(=8.91$ $\mathrm{Mg} / \mathrm{m}^{3}$ ). A scanning electron microscope (SEM) was used to characterize the structure of the $\mathrm{Ni}$ DG. Nanoindentation experiments were performed using a Hysitron Triboindenter equipped with a diamond sphero-conical tip of semi-conical angle $\alpha=30^{\circ}$, and a spherical tip of radius $R=5.07$ $\mu \mathrm{m}$. Tests were performed in load control, with fixed loading and unloading rates of $40 \mu \mathrm{N} / \mathrm{s}$ and a hold time of $2 \mathrm{~s}$ at peak load $P_{\max }$, where $P_{\max }$ equals $200,500,1000$ and $1500 \mu \mathrm{N}$. A total of 5 indentations were performed at each value of $P_{\max }$. The limiting depth of penetration, $h_{\text {lim }}$, up to which depth the indentation can be considered spherical, is given as $h_{\text {lim }}=R(1-\sin \alpha)$ [16], which gives $h_{\text {lim }}=2.5 \mu \mathrm{m}$. Since the depth of penetration at peak load $h_{\max }$ does not exceed $200 \mathrm{~nm}$, the indentations can be considered spherical. Note also that this value of $h_{\max }$ is about one-tenth of the film thickness and hence satisfies the guideline that $h_{\max }$ should be less than $10 \%$ of film thickness in order for the substrate to not affect the measured indentation response [17].

The widely used Oliver-Pharr method of deducing the material properties $E$ and $H$ from the load versus depth of penetration $(P-h)$ curves was developed for incompressible solids and for the case where the size of the indent is not known from direct visual observation. In the present study, Ni DG is highly compressible, and the size of indenter imprints is known from SEM measurements. Consequently, $H$ is obtained directly as:

$$
H=\frac{P_{\max }}{A_{t}}
$$

where $A_{t}$ is the projected contact area $\left(=\pi a^{2}\right.$, where $a$ is the radius of the circular imprint). The reduced elastic modulus, $E_{r}$, was determined from the $P-h$, responses using the Sneddon [18] formula for a flat-bottomed, frictionless punch:

$$
E_{r}=\frac{M}{2} \frac{\sqrt{\pi}}{\sqrt{A_{t}}}
$$


where $M$ is the slope of the unloading curve at $h_{\max }$. The modulus of the Ni DG sample, $E$ was then obtained from the standard relation for the reduced modulus:

$$
\frac{1}{E_{r}}=\frac{1-v_{i}^{2}}{E_{i}}+\frac{1-v^{2}}{E}
$$

where $E_{i}$ and $v_{i}$ are the modulus and Poisson's ratio of the indenter, which are taken as $1140 \mathrm{GPa}$ and 0.07 , respectively, while the Poisson's ratio of the Ni DG is taken to be $v=0.35$ [15]. The average indentation strain $\varepsilon_{i}$ is defined in [19] by

$$
\varepsilon_{i}=0.2 \mathrm{a} / \mathrm{R}
$$
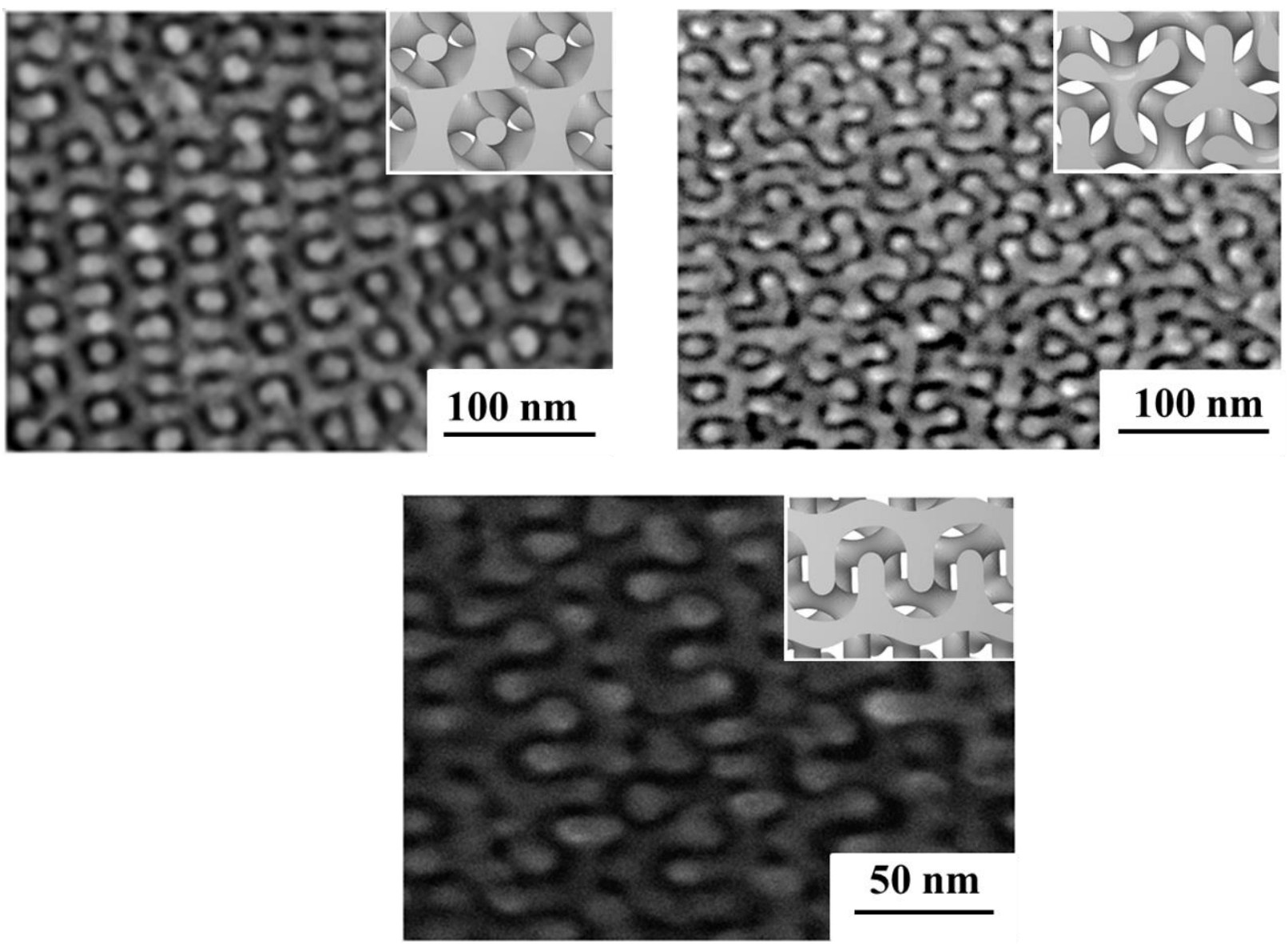

Figure 1. SEM images showing characteristic (a) (110), (b) (111) and (c) (211) planes of DG structure. Simulated structures of the corresponding planes are displayed in respective insets. 
Representative SEM images of different lattice planes of the Ni DG are shown in Fig. 1. These images indicate that the DG structure is uniform, with an inter-percolating structure of ligaments and pores. The strut diameter and length were estimated from such images as $10.8 \pm 0.3$ and $31.1 \pm 1.8 \mathrm{~nm}$, respectively. The diameter of the pores is similar to that of the strut diameter. Representative load versus displacement, $P$ vs. $h$, responses are shown in Fig. 2 (a) for selected values of $P_{\max }$. Both the loading and unloading segments of the $P$ - $h$ curves are devoid of serrations or pop-ins, implying that plastic deformation is smooth, with no evidence of cracking or delamination between the DG film and the substrate. Note also that a significant residual indent exists after unloading even in the case of $P_{\max }$ of $200 \mu \mathrm{N}$, which corresponds to a value of $\varepsilon_{\mathrm{i}}$ equal to $1.5 \%$, implying that plastic deformation initiates at low strains.
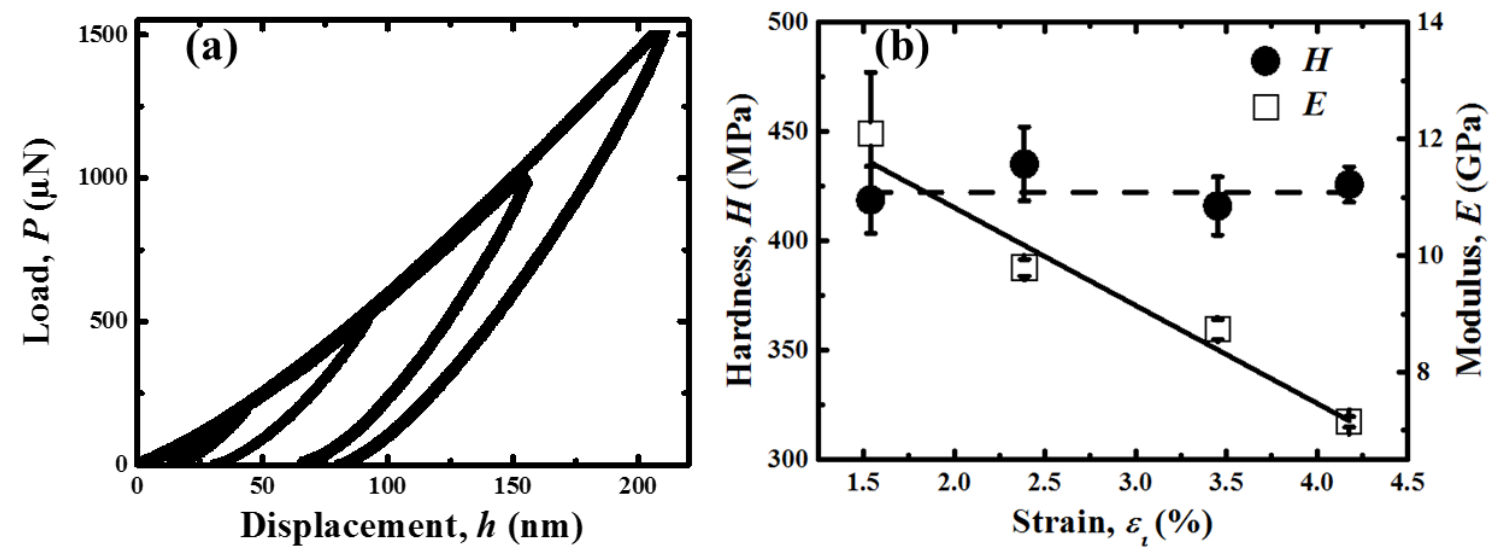

Figure 2. (a) Representative load $P$ versus depth of penetration $h$ response for selected values of maximum load, $\mathrm{P}_{\max }$. (b) Hardness $H$ and modulus $E$ of the film versus indentation strain $\varepsilon_{i}$.

The dependence of $H$ and $E$ upon $\varepsilon_{\mathrm{i}}$ is plotted in Fig. 2 (b). While $H$ is invariant, $E$ decreases linearly with increasing $\varepsilon_{\mathrm{i}}$, and has an intercept of $E_{0}=14.2 \pm 0.9 \mathrm{GPa}$ at $\varepsilon_{\mathrm{i}}=0$. This value of Young's modulus $E_{0}$ for the Ni DG in the pristine state of $\varepsilon_{\mathrm{i}}=0$ is similar to the value of $15 \mathrm{GPa}$ as determined by Khaderi et al. [15] in their simulations and experiments.

Khaderi et al. [20] predicted that the Young's modulus $E^{D G}$ and yield strength $\sigma_{y}^{D G}$ of the DG is related to the respective parent properties $E^{s}$ and $\sigma_{\mathrm{y}}^{\mathrm{s}}$ of cell wall material according to:

$$
\begin{aligned}
& E^{D G}=0.43 E^{s}\left(\rho_{r}\right)^{2} \\
& \sigma_{y}^{D G}=0.44 \sigma_{\mathrm{y}}^{\mathrm{s}}\left(\rho_{r}\right)^{1.5}
\end{aligned}
$$


where $E^{S}$ and $\sigma_{\mathrm{y}}^{\mathrm{s}}$ are the elastic modulus and yield strength, respectively, of the fully dense cell wall material. Upon substituting the measured values of $E^{D G}=E_{0}=14.2 \pm 0.9 \mathrm{GPa}$ and $\rho_{\mathrm{r}}=0.40$ into eq. (5), we predict that $E^{s}=208 \pm 14 \mathrm{GPa}$, which is in excellent agreement with the textbook value of 200 - $220 \mathrm{GPa}$ for the Young's modulus of Ni [21].

The hardness of a Ni DG lattice can be directly related to the compressive yield strength, $\sigma_{y}^{D G}$ of the Ni DG lattice via a constraint factor, $C$. Recall that, for a fully dense metallic alloy, it is usual to take $C=3$, whereas, for a highly compressible solid, $C$ is close to unity [22]. Upon assuming $C=1$ for the Ni DG lattice, the uniaxial compressive strength equals the hardness $H$, and consequently $\sigma_{y}^{D G}=424 \pm 8 \mathrm{MPa}$. Now make use of eq. (6) to obtain $\sigma_{\mathrm{y}}^{\mathrm{s}}=3.82 \pm 0.08 \mathrm{GPa}$ for the Ni cell walls. This value is substantially larger than the yield strength of bulk and polycrystalline pure $\mathrm{Ni}$, which are well below $150 \mathrm{MPa}$ for a grain size in the range 0.1 to $130 \mu \mathrm{m}$ [23].

We emphasize that the inferred value of $\sigma_{\mathrm{y}}^{\mathrm{s}}$ corresponds to the yield strength of an individual strut. Since the strut diameter is only $10 \mathrm{~nm}$, it is instructive to compare the value of $\sigma_{\mathrm{y}}^{\mathrm{s}}$ $=3.8 \mathrm{GPa}$ with the data obtained for pure $\mathrm{Ni}$ of similar characteristic length scale. A yield strength of $\sigma_{\mathrm{y}}^{\mathrm{s}}=2 \mathrm{GPa}$ can be deduced from the indentation tests of Schuh et al. [24] and similar studies $[25,26]$ on nanocrystalline $\mathrm{Ni}$ (average grain size of $12 \mathrm{~nm}$ ), upon assuming a constraint factor of $C=3$ in their tests. Likewise, uniaxial compression tests on single crystal micro-pillars of $\mathrm{Ni}$ reveal that the flow stress at 3\% true strain is approximately $1.6 \mathrm{GPa}$ for pillars of diameter 165 $\mathrm{nm}$ [27]. An extrapolation of such micro-pillar data (for example Fig. 4 of ref. [27]) to sub-100 nm pillar diameters implies a yield strength of $2 \mathrm{GPa}$.

The inferred strength of the Ni cell walls in the DG lattice, $\sigma_{\mathrm{y}}^{\mathrm{s}}=3.8 \mathrm{GPa}$, is about half the ideal strength, $G_{\mathrm{s}} / 10$, where $G_{\mathrm{s}}$ is the shear modulus of fully dense Ni (76 GPa); our inferred strength of $\sigma_{\mathrm{y}}^{\mathrm{s}}=3.8 \mathrm{GPa}$ is similar to that of Khaderi et al. [15] $\left(\sigma_{\mathrm{y}}^{\mathrm{s}}=5.7 \mathrm{GPa}\right)$ on the basis of a Berkovich nanoindentation study. Khaderi et al. [15] reasoned that the low dislocation content in struts of small diameter $(\sim 10 \mathrm{~nm})$ is responsible for the extremely high strength of the pure Ni.

The observed insensitivity of hardness $H$ to the representative indentation strain $\varepsilon_{\text {i }}$ suggests that the plastic collapse of the Ni struts, followed by densification of the DG, occurs at low $\varepsilon_{\mathrm{i}}$ and remains the dominant mechanism thereafter [20]. This conclusion is supported by the postindentation imaging of the imprints; SEM images of the imprint made at different $\mathrm{P}_{\max }$ (or $\varepsilon_{\mathrm{i}}$ ) are 
displayed in Fig. 3. The approximate areas of residual impressions are marked with the dotted circles. For example, the diameter of the impression was found to be $1.75 \mu \mathrm{m}$ at $\varepsilon_{\mathrm{i}}=3.5 \%$. Broadly, the indentation images are in agreement with the expectation of plastic collapse of the struts and densification of the cellular DG underneath the indenter [28-30]. The nanolattice remains agglomerated after release of the indent load due to the drop in surface energy associated with flocculation [31,32]. It is interesting to note that the indents made at $P_{\max }=500,1000$ and 1500 $\mu \mathrm{N}$ displayed in Fig. $3(\mathrm{~b}-\mathrm{d})$ consist of islands of consolidated material, which are connected to each other by isolated ligaments. These consolidated islands appear to have fragmented from a larger mass into irregular shapes that are not larger than $\sim 0.5 \mu \mathrm{m}$ in size.

We believe that the linear decrease in $E$ with increasing $\varepsilon_{\mathrm{i}}$ is related to the above mentioned progressive plastic collapse and densification of the struts into islands. The estimation of $E$ depends on the accurate measurement of contact area and $M$ (see eq. 2). In our study the contact area has been approximated as $A_{\mathrm{t}}$, which is the projected area that is within the indenter impressions (and estimated using the circles drawn) displayed in Fig. 3. The implicit assumption here is that the structure and topology of the material does not change during indentation. However, as noted in Fig. 3, the DG structure under the indenter transforms to interconnected fused islands as $\varepsilon_{i}$ increases. Since the effective surface area of the fused struts in the indented volume is lower than that of the DG structure in its pristine state, it is likely that $A_{\mathrm{t}}$ could have been overestimated. This implies that the value of $E$, which is proportional to $1 /\left(A_{\mathrm{t}}\right)^{1 / 2}$, may have been underestimated. Using image analysis, we calculated only the projected areas of the fused masses, $A_{\mathrm{f}}$, and used this the contact area for different $P_{\max }$ to re-calculate $E$. The original and corrected values of $E$ along with $A_{\mathrm{t}}$ and $A_{\mathrm{f}}$ are listed in Table 1 . The values of corrected $E \sim 11 \mathrm{GPa}$, within error, at all $P_{\max }$, except at $200 \mu \mathrm{N}$, where the indented area undergoes complete densification. This implies that the variations in $E$ is influenced by the measurement of the contact area. Another source of variations in $E$ could be an artifact associated with the measurement of $M$ during fusion of the struts. Although it is not clear when the struts fuse during indentation, the subsequent fragmentation of the fused masses can reduce the recovery of the material during unloading. As $\varepsilon_{\mathrm{i}}$ increases, a higher degree of fragmentation occurs that leads to a progressive reduction in $M$, and by extension $E$. However, these hypotheses can be confirmed only after further, in situ experiments are conducted. 


\begin{tabular}{|l|l|l|l|l|}
\hline$P_{\max }(\mu \mathrm{N})$ & $\begin{array}{l}\text { Projected contact } \\
\text { area of indent, } A_{\mathrm{t}} \\
\left(\mathrm{nm}^{2}\right)\end{array}$ & $\begin{array}{l}\text { Uncorrected } \\
(\mathrm{GPa})\end{array}$ & $\begin{array}{l}\text { Projected area of } \\
\text { fused islands, } A_{\mathrm{f}} \\
\left(\mathrm{nm}^{2}\right)\end{array}$ & $\begin{array}{l}\text { Corrected } \\
(\mathrm{GPa})\end{array}$ \\
\hline 200 & 477836.2 & 12.08 & 477836.2 & 12.08 \\
\hline 500 & 1149901.5 & 9.8 & 862426.1 & 11.2 \\
\hline 1000 & 2405281.9 & 8.73 & $1,866,498.7$ & 10.94 \\
\hline 1500 & 3523236.5 & 7.14 & $1,713,941.9$ & 10.20 \\
\hline
\end{tabular}

Table 1. Summary of projected area measurements from image analysis and the corrected value of elastic modulus, $E$.
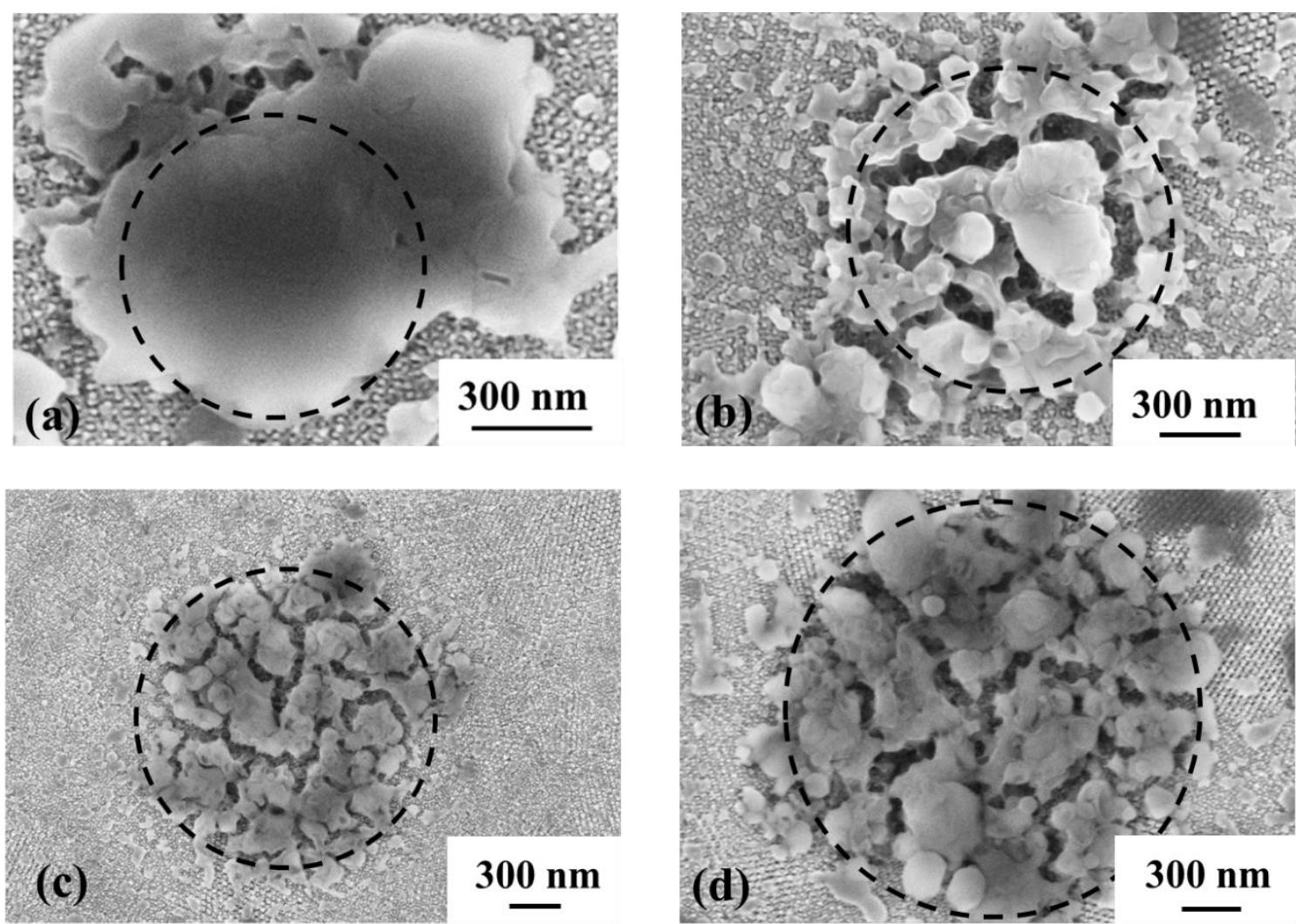

Figure 3: SEM images of the residual impressions obtained after indentation tests with $P_{\max }$ of (a) $200 \mu \mathrm{N}$, (b) $500 \mu \mathrm{N}$, (c) $1000 \mu \mathrm{N}$ and (d) $1500 \mu \mathrm{N}$. The dashed circles represents the approximate projected contact area.

In summary, nanoindentation experiments employing a spherical tip indentation were performed on a Ni DG nanolattice material, so as to assess its hardness, and elastic modulus as a function of strain, which the spherical tipped indenter facilitates. Images of the indenter impressions confirm that plastic collapse, followed by densification, of the cellular structure occurs 
readily at indentation strains as low as $\sim 1.5 \%$. The hardness and extrapolated modulus to zero strain were used to extract the properties of individual struts using the analytical expressions available in literature. The extracted modulus is in excellent agreement with the Young's modulus of bulk Ni. The estimated yield strength of the struts is more than a magnitude higher than that of coarse-grained polycrystalline $\mathrm{Ni}$ and nearly-double that reported for nanocrystalline Ni and nanopillars of single crystal $\mathrm{Ni}$, but somewhat smaller than that estimated using Berkovich tip nanoindentation experiments on Ni DG. The extremely high strength of the struts is attributed to the nm-scale dimensions of the DG's struts and to their defect-free nature.

\section{References}

[1] E.J.W. Crossland, M. Kamperman, M. Nedelcu, C. Ducati, U. Wiesner, D.-M. Smilgies, G.E.S. Toombes, M.A. Hillmyer, S. Ludwigs, U. Steiner, H.J. Snaith, Nano Lett. 9 (2009) 2807-2812.

[2] I. Vukovic, G. ten Brinke, K. Loos, Polymer 54 (2013) 2591-2605.

[3] M.R.J. Scherer, U. Steiner, Nano Lett. 13 (2013) 3005-3010.

[4] H.-Y. Hsueh, Y.-C. Huang, R.-M. Ho, C.-H. Lai, T. Makida, H. Hasegawa, Adv. Mater. 23 (2011) 3041-3046.

[5] M.R.J. Scherer, L. Li, P.M.S. Cunha, O.A. Scherman, U. Steiner, Adv. Mater. 24 (2012) 1217-1221.

[6] I. Vukovic, S. Punzhin, Z. Vukovic, P. Onck, J.Th.M. De Hosson, G. ten Brinke, K. Loos, ACS Nano 5 (2011) 6339-6348.

[7] E. Detsi, S.H. Tolbert, S. Punzhin, J.Th.M. De Hosson, J. Mater. Sci. 51 (2016) 615-634.

[8] W.-H. Lin, Y. Teng, Z.-D. Sha, S.-Y. Yuan, P.S. Branicio, Int. J. Plast. (2020) 102657.

[9] I.-C. Cheng, A.M. Hodge, Scr. Mater. 69 (2013) 295-298.

[10] D. Farkas, A. Caro, E. Bringa, D. Crowson, Acta Mater. 61 (2013) 3249-3256.

[11] J.R. Greer, J.Th.M. De Hosson, Prog. Mater. Sci. 56 (2011) 654-724.

[12] J.R. Greer, W.C. Oliver, W.D. Nix, Acta Mater. 53 (2005) 1821-1830.

[13] V.S. Deshpande, M.F. Ashby, N.A. Fleck, Acta Mater. 49 (2001) 1035-1040.

[14] X.-Y. Sun, G.-K. Xu, X. Li, X.-Q. Feng, H. Gao, J. Appl. Phys. 113 (2013) 023505.

[15] S.N. Khaderi, M.R.J. Scherer, C.E. Hall, U. Steiner, U. Ramamurty, N.A. Fleck, V.S. Deshpande, Extreme Mech. Lett. 10 (2017) 15-23.

[16] Z. Cao, X. Zhang, Thin Solid Films 516 (2008) 1941-1951.

[17] A.C. Fischer-Cripps, Nanoindentation, Springer, 2011.

[18] I.N. Sneddon, Math. Proc. Camb. Philos. Soc. 44 (1948) 492-507.

[19] E.G. Herbert, G.M. Pharr, W.C. Oliver, B.N. Lucas, J.L. Hay, Thin Solid Films 398-399 (2001) 331-335.

[20] S.N. Khaderi, V.S. Deshpande, N.A. Fleck, Int. J. Solids Struct. 51 (2014) 3866-3877.

[21] H.E. Boyer, ASM Int. Met. Park Ohio 44073 USA 1987630 (1987).

[22] N.A. Fleck, H. Otoyo, A. Needleman, Int. J. Solids Struct. 29 (1992) 1613-1636.

[23] A.A.W. Thompson, Acta Metall. 23 (1975) 1337-1342.

[24] C.A. Schuh, T.G. Nieh, T. Yamasaki, Scr. Mater. 46 (2002) 735-740. 
[25] R. Mishra, B. Basu, R. Balasubramaniam, Mater. Sci. Eng. A 373 (2004) 370-373.

[26] N.P. Wasekar, P. Haridoss, S.K. Seshadri, G. Sundararajan, Surf. Coat. Technol. 291 (2016) 130-140.

[27] C.P. Frick, B.G. Clark, S. Orso, A.S. Schneider, E. Arzt, Mater. Sci. Eng. A 489 (2008) 319-329.

[28] N.J. Briot, T.J. Balk, MRS Commun. 8 (2018) 132-136.

[29] A. Leitner, V. Maier-Kiener, J. Jeong, M.D. Abad, P. Hosemann, S.H. Oh, D. Kiener, Acta Mater. 121 (2016) 104-116.

[30] J. Biener, A.M. Hodge, A.V. Hamza, Appl. Phys. Lett. 87 (2005) 121908.

[31] M.G. McKimpson, Mater. Manuf. Process. 11 (1996) 935-949.

[32] P.A. Kralchevsky, N.D. Denkov, Curr. Opin. Colloid Interface Sci. 6 (2001) 383-401. 